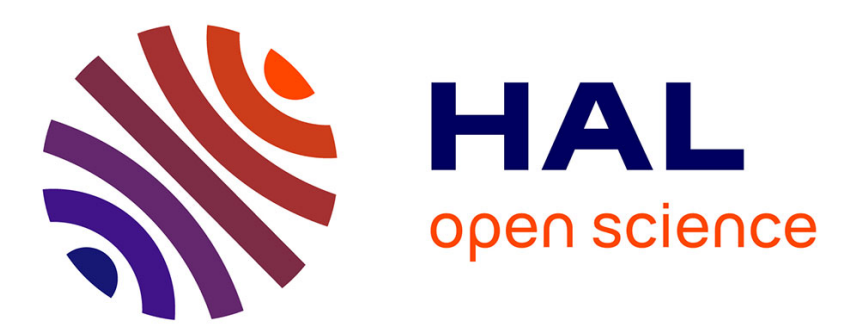

\title{
Least costly identification experiment for control
}

Xavier Bombois, Gérard Scorletti, Michel Gevers, Paul van den Hof, Roland Hildebrand

\section{To cite this version:}

Xavier Bombois, Gérard Scorletti, Michel Gevers, Paul van den Hof, Roland Hildebrand. Least costly identification experiment for control. Automatica, 2006, 42 (10), pp.1651-1662. 10.1016/j.automatica.2006.05.016 . hal-00413370

\section{HAL Id: hal-00413370 https://hal.science/hal-00413370}

Submitted on 8 Jan 2014

HAL is a multi-disciplinary open access archive for the deposit and dissemination of scientific research documents, whether they are published or not. The documents may come from teaching and research institutions in France or abroad, or from public or private research centers.
L'archive ouverte pluridisciplinaire HAL, est destinée au dépôt et à la diffusion de documents scientifiques de niveau recherche, publiés ou non, émanant des établissements d'enseignement et de recherche français ou étrangers, des laboratoires publics ou privés. 


\title{
Least costly identification experiment for control
}

\author{
X. Bombois ${ }^{a}$, G. Scorletti, ${ }^{\mathrm{b}} \mathrm{M}$. Gevers, ${ }^{\mathrm{c}}$ P.M.J. Van den Hof ${ }^{\mathrm{a}}$ and R. Hildebrand ${ }^{\mathrm{d}}$ \\ ${ }^{a}$ Delft Center for Systems and Control, Delft University of Technology, Delft, The Netherlands \\ ${ }^{\mathrm{b}}$ GREYC-Equipe AUTO, Caen, France \\ ${ }^{\mathrm{c}}$ CESAME, Louvain-la-Neuve, Belgium \\ ${ }^{\mathrm{d}} I M A G$, Grenoble, France
}

\begin{abstract}
All approaches to optimal experiment design for control have so far focused on deriving an input signal (or input signal spectrum) that minimizes some control-oriented measure of plant/model mismatch between the nominal closed loop system and the actual closed loop system, typically under a constraint on the total input power. In practical terms, this amounts to finding the (constrained) input signal that minimizes a measure of a control-oriented model uncertainty set. Here we address the experiment design problem from a "dual" point of view and in a closed-loop setting: given a maximum allowable controloriented model uncertainty measure compatible with our robust control specifications, what is the cheapest identification experiment that will give us an uncertainty set that is within the required bounds? The identification cost can be measured by either the experiment time, the performance degradation during experimentation due to the added excitation signal, or a combination of both. Our results are presented for the situation where the control objective is disturbance rejection only.
\end{abstract}

Key words: identification for control, experiment design.

\section{Introduction}

The problem addressed and solved in this paper is a novel formulation of an optimal experiment design problem, in the context of "identification for robust control". It is novel essentially in that it takes the dual viewpoint to the classical way of posing optimal experiment design problems. To state this in a nutshell, the classical way is to seek the optimal input signal (or the optimal input signal spectrum) that minimizes some control-oriented measure of the quality of the identified model, subject to constraints on the input signal power (and/or on the output signal power). Representative examples of such approach can be found in $[10,12,17,9,16,6,11]$. From a practical point of view [20], such approach is not always the most sensible choice: one should not spend more effort on the identification than what is needed to achieve the required robust control performance.

Thus, here we consider a "dual approach" to this optimal experiment design problem. Given some prior bounds on the uncertainty around an identified model that is

Email address: x.j.a.bombois@dcsc.tudelft.nl (X. Bombois). compatible with the robust control performance specifications when such model is to be used for robust control design, we seek to minimize the identification cost such that the uncertainty set around the identified model is (just) within these prior bounds. Initial steps in the direction of minimizing the input effort given a required quality measure have been taken in [4], where open-loop identification was considered with a constraint on the total input power, together with an $H_{\infty}$ robust control design criterion. The idea of minimizing the total input power for open-loop identification, subject to achieving a required quality measure on the estimated model, has been further developed in [14], where a variety of quality measures has been considered. Here we solve this optimal experiment design problem in a closed-loop setup.

The problem formulation addressed in this paper is as follows. We consider that an unknown "true" linear timeinvariant system $G_{0}$ is controlled with an existing controller $C_{i d}$ that is not fully satisfactory, and which one wants to replace by a new controller. The control objective is regulation only, i.e. the normal operation of the closed-loop system is with a constant reference signal, which, for simplicity, we assume to be zero. For the purpose of designing a new and robust controller, an identi- 
fication step is performed in closed loop with the existing controller $C_{i d}$, leading to a model $\hat{G}$ of the unknown system $G_{0}$ and a parametric uncertainty region $\mathcal{D}$ centered around $\hat{G}$ and containing the true system $G_{0}$ with some pre-chosen probability, say $\beta[17,1]$.

A new controller $\hat{C}(\hat{G})$ will be designed from this identified model $\hat{G}$ using a pre-defined control design method (e.g. an $H_{\infty}$ control design method with fixed weights). The requirement imposed on the new controller is that it achieves a sufficient $H_{\infty}$ performance with the true system $G_{0}$. However, $G_{0}$ is unknown and therefore $\hat{C}(\hat{G})$ is designed in such a way that it achieves this sufficient level of $H_{\infty}$ performance for a set of systems $\mathcal{D}_{a d m}$ around the estimated $\hat{G}$ (the set $\mathcal{D}_{a d m}$ will be formally defined at the end of Section 3). Returning now to the identification part of the exercise, the whole idea of our optimal experiment design program is to perform a closed-loop identification experiment with the smallest possible cost so that the uncertainty set $\mathcal{D}$ around the estimated $\hat{G}$ lies within the admissible uncertainty set $\mathcal{D}_{a d m}$ imposed by the control performance specifications. This implies, in particular, that the true system $G_{0}$, which lies within $\mathcal{D}$ with probability $\beta$, will also lie in $\mathcal{D}_{a d m}$ with probability at least equal to $\beta$, and will therefore achieve the required $H_{\infty}$ performance level with $\hat{C}(\hat{G})$ with the same probability. The size of the identified $\mathcal{D}$ is a function of the covariance matrix $P_{\theta}$ of the identified parameter vector $\hat{\theta}_{N}$ and, consequently, a function of the length $N$ of the identification experiment and of the power spectrum $\Phi_{r}(\omega)$ of the excitation signal $r(t)$ used for the identification (see Figure 1).

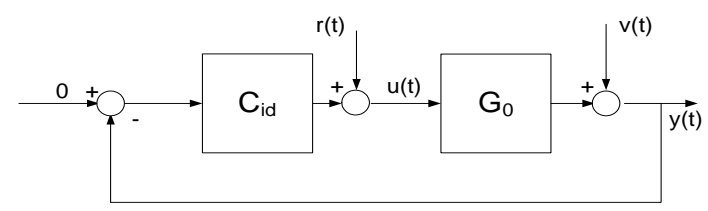

Fig. 1. Closed loop $\left[C_{i d} G_{0}\right]$ during an identification experiment with $r(t)$ as excitation signal.

It remains to specify what is meant by "identification with the smallest possible cost". In the present context of a regulation problem, any external excitation signal $r$ that is added for the purpose of identifying the system produces added perturbations $y_{r}$ and $u_{r}$ to the normal operating signals $y$ and $u$. During the experiment time, these perturbations increase the variance of $y$ and $u$ beyond their normal value, and consequently cause a degradation of the production quality. It is this degradation that we shall seek to minimize while achieving the required precision $\left(\mathcal{D} \subseteq \mathcal{D}_{a d m}\right)$.

All results in this paper are derived under the assumption that the system is in the model set, which implies that we consider variance errors only. However, unlike $[10,12,9]$, our results are not based on the asymptotic (in model order) transfer function variance expressions [17][page 295], but on the more accurate parameter covariance expressions for finite model orders, that were also used in [11].

Our first contribution, based on a classical result [21][page 392], is to show that, when the controller $C_{i d}$ is sufficiently complex, the model accuracy required for any robust performance specifications can be achieved at no cost at all, provided that one can wait long enough, i.e. no external excitation signal $r$ is required provided the data length $N$ can be made sufficiently large. The minimal data length required to achieve the desired model accuracy can be determined via an LMI optimization. Such a strategy is not always practically feasible. Indeed, the existing controller $C_{i d}$ may e.g. be of insufficient order or one might have to wait an unduly long time to get the required accuracy. Thus, we shall present a number of optimal experiment design scenarios, where the optimal design will be a trade-off between data length and a sufficient amount of excitation power $\Phi_{r}(\omega)$. This is done by providing an LMI-based solution to the following two experiment design problems:

- For a given excitation spectrum $\Phi_{r}(\omega)$, determine the minimal experiment time $N$ that is necessary to meet the robust performance constraint, i.e. $\mathcal{D} \subseteq \mathcal{D}_{a d m}$.

- For a given experiment time $N$, determine the power spectrum $\Phi_{r}(\omega)$ which induces the smallest perturbation with respect to the normal operating conditions of the control loop $\left[C_{i d} G_{0}\right]$, and which achieves the required robust performance constraints, i.e. $\mathcal{D} \subseteq \mathcal{D}_{a d m}$.

Our tools for cheap identification for robust control are developed for direct closed-loop Prediction Error identification [21][page 389]. The open-loop case was treated in [3].

\section{Prediction Error Identification aspects}

We consider the identification of a stable linear timeinvariant single input single output system with a model structure $\mathcal{M}=\{G(z, \theta), H(z, \theta)\}, \theta \in \mathbf{R}^{k}$, that is able to represent the true system. Thus, the finitedimensional true system is given by:

$\mathcal{S}: y(t)=\overbrace{G\left(z, \theta_{0}\right)}^{G_{0}(z)} u(t)+\overbrace{H\left(z, \theta_{0}\right) e(t)}^{v(t)}$

for some unknown parameter vector $\theta_{0} \in \mathbf{R}^{k}$. In (1), $e(t)$ is a white noise with variance $\sigma_{e}^{2}$ and $G\left(z, \theta_{0}\right), H\left(z, \theta_{0}\right)$ are stable discrete-time transfer functions. The transfer function $H\left(z, \theta_{0}\right)$ is furthermore assumed monic and minimum-phase. We shall also assume throughout the paper that the model structure is globally identifiable at $\theta_{0}$. This means that $\theta_{0}$ is the only value of $\theta$ for which $G(z, \theta)$ and $H(z, \theta)$ represent the true system (see Theorem 4.2 of [17]). 
This true system is operated in closed loop with an initial (but unsatisfactory) controller $C_{i d}: u(t)=r(t)-$ $C_{i d}(z) y(t)$. The signal $r(t)$ is zero in normal operation but can be used to excite the system for a closed-loop identification experiment (see Figure 1). With $S_{i d} \triangleq$ $1 /\left(1+C_{i d} G_{0}\right)$, the closed-loop system can be written as:

$$
\begin{aligned}
& y(t)=S_{i d} v(t)+\overbrace{G_{0} S_{i d} r(t)}^{y_{r}(t)} \\
& u(t)=-C_{i d} S_{i d} v(t)+\underbrace{S_{i d} r(t)}_{u_{r}(t)}
\end{aligned}
$$

Suppose it is desired to identify a model $\hat{G}(z)=$ $G\left(z, \hat{\theta}_{N}\right), \hat{H}(z)=H\left(z, \hat{\theta}_{N}\right)$ of the true system by applying an external signal $r$ to the actual closed-loop system with the controller $C_{i d}$ in the loop and by collecting $N$ input-output data, using a direct Prediction Error (PE) identification method. We shall assume throughout this paper that the signal $r$ is taken as a part of length $N$ of a realization of a quasi-stationary signal with power spectrum $\Phi_{r}(\omega)$, yielding measured signals $\{u(t), y(t), t=1, \ldots, N\} . \Phi_{r}(\omega)$ is defined as the Fourier transform of $R_{r}(\tau)=\lim _{N \rightarrow \infty} \frac{1}{N} \sum_{t=1}^{N} \operatorname{Er}(t) r(t-\tau)$. The estimated parameter vector $\hat{\theta}_{N}$ is then defined by: $\hat{\theta}_{N} \triangleq \arg \min _{\theta} \frac{1}{N} \sum_{t=1}^{N} \epsilon^{2}(t, \theta)$ with $\epsilon(t, \theta) \triangleq$ $H(z, \theta)^{-1}(y(t)-G(z, \theta) u(t))$. Note that $\epsilon(t, \theta)$ depends on the chosen signal $r(t)$ via (2)-(3).

In this paper, as is usual in PE identification, the experimental conditions will be chosen/designed such that the closed-loop identification experiment is "informative enough" [17] i.e. such that the cost function:

$\bar{V}(\theta)=\bar{E} \epsilon^{2}(t, \theta)=\lim _{N \rightarrow \infty} \frac{1}{N} \sum_{t=1}^{N} E \epsilon^{2}(t, \theta)$

has a unique minimum (to which $\hat{\theta}_{N}$ tends w.p. 1 when $N \rightarrow \infty)$. By our standing assumption that the system is in the model set and that the model structure is globally identifiable at $\theta_{0}$, this unique minimum is $\theta_{0}$. The informative character of the experiment depends on the complexity of the controller $C_{i d}$ (see Section 5) and can always be guaranteed by proper choice of $\Phi_{r}(\omega)$. Indeed, a signal $r(t)$ generated by filtering a white noise with a finite-dimension filter will always lead to an informative enough experiment. See e.g. [17][page 427] for details. Under these conditions, we have the following important result.

Lemma 1 Consider a closed-loop identification experiment, as described above, that is informative enough. Then the identified parameter vector $\hat{\theta}_{N}$ is asymptotically normally distributed around the true parameter vector $\theta_{0}$, i.e. $\hat{\theta}_{N} \sim \mathcal{N}\left(\theta_{0}, P_{\theta}\right)$ with $P_{\theta}$ a strictly positive definite matrix given by [17][Chapter 9]:

$P_{\theta}=\frac{\sigma_{e}^{2}}{N}\left(\bar{E}\left(\psi\left(t, \theta_{0}\right) \psi\left(t, \theta_{0}\right)^{T}\right)\right)^{-1}$ with $\psi(t, \theta)=-\frac{\partial \epsilon(t, \theta)}{\partial \theta} .(5)$

In this paper, the design variables with respect to which we shall optimize the identification experiment are the data length $N$, and the external signal $r$ via its spectrum $\Phi_{r}(\omega)$. The following expression, which is easily deduced from (3) and (5), shows precisely how the covariance matrix $P_{\theta}$ in $(5)$ depends on these design variables:

$$
\begin{aligned}
& P_{\theta}^{-1}=N \overbrace{\left(\frac{1}{\sigma_{e}^{2}} \frac{1}{2 \pi} \int_{-\pi}^{\pi} F_{r}\left(e^{j \omega}, \theta_{0}\right) F_{r}\left(e^{j \omega}, \theta_{0}\right)^{*} \Phi_{r}(\omega) d \omega\right)}^{\mathcal{P}_{r}^{-1}\left(\Phi_{r}(\omega), \theta_{0}, \sigma_{e}^{2}\right)} \\
&+N \underbrace{\left(\frac{1}{2 \pi} \int_{-\pi}^{\pi} F_{e}\left(e^{j \omega}, \theta_{0}\right) F_{e}\left(e^{j \omega}, \theta_{0}\right)^{*} d \omega\right)}_{\mathcal{P}_{v}^{-1}\left(\theta_{0}\right)}
\end{aligned}
$$

Here, $F_{r}\left(z, \theta_{0}\right)=S_{i d} \frac{\Lambda_{G}\left(z, \theta_{0}\right)}{H\left(z, \theta_{0}\right)}, F_{e}\left(z, \theta_{0}\right)=\frac{\Lambda_{H}\left(z, \theta_{0}\right)}{H\left(z, \theta_{0}\right)}-$ $C_{i d} S_{i d} \Lambda_{G}\left(z, \theta_{0}\right), \Lambda_{G}(z, \theta)=\frac{\partial G(z, \theta)}{\partial \theta}$ and $\Lambda_{H}(z, \theta)=$ $\frac{\partial H(z, \theta)}{\partial \theta}$.

Using Lemma 1, it is possible to define an uncertainty region $\mathcal{D}\left(\hat{\theta}_{N}, P_{\theta}\right)$ around the identified model and containing the unknown true system $G\left(z, \theta_{0}\right)$ at any desired probability level $\beta[1]$ :

$$
\begin{aligned}
\mathcal{D}\left(\hat{\theta}_{N}, P_{\theta}\right)= & \left\{G(z, \theta)=\frac{Z_{N}(z) \theta}{1+Z_{D}(z) \theta} \mid \theta \in U,\right. \\
& \left.U=\left\{\theta \mid\left(\theta-\hat{\theta}_{N}\right)^{T} P_{\theta}^{-1}\left(\theta-\hat{\theta}_{N}\right)<\chi\right\}\right\}
\end{aligned}
$$

where $\chi$ is a real constant dependent on the chosen probability level $\beta$ and $Z_{N}, Z_{D}$ are row vectors containing delays and zeros. These vectors introduce a general parametrization of $G(z, \theta)$ which will be important in the sequel. Note that the size of the uncertainty region $\mathcal{D}\left(\hat{\theta}_{N}, P_{\theta}\right)$ is a function of the covariance matrix $P_{\theta}$ and thus, by (6), a function of the design variables $N$ and $\Phi_{r}(\omega)$.

\section{Control design objectives and control design method}

As stated before, our aim is to replace the "unsatisfactory" controller $C_{i d}(z)$ by a "satisfactory" controller $\hat{C}(z)$. In order to define what we mean by satisfactory controller, we adopt the following performance measure for a stable loop $[C G]$ :

$$
J\left(G, C, W_{l}, W_{r}\right)=\sup _{\omega} \bar{J}\left(\omega, G, C, W_{l}, W_{r}\right)
$$


with

$\bar{J}\left(\omega, G, C, W_{l}, W_{r}\right)=\bar{\sigma}\left(W_{l}\left(e^{j \omega}\right) F\left(G\left(e^{j \omega}\right), C\left(e^{j \omega}\right)\right) W_{r}\left(e^{j \omega}\right)\right)(9)$

$F(G, C) \triangleq\left(\begin{array}{cc}\frac{G C}{1+G C} & \frac{G}{1+G C} \\ \frac{C}{1+G C} & \frac{1}{1+G C}\end{array}\right)$

where $\bar{\sigma}(A)$ denotes the largest singular value of $A$ and $W_{l}(z), W_{r}(z)$ are chosen diagonal performance filters. The performance measure (8) is quite general: $J\left(G, C, W_{l}, W_{r}\right) \leq 1$ ensures that the four entries of $W_{l}(z) F(G, C) W_{r}(z)$ have an $H_{\infty}$ norm smaller than one. Simpler $H_{\infty}$ criteria can be chosen as special cases; e.g., for $W_{l}(z)=\operatorname{diag}(0, W(z))$ and $W_{r}=\operatorname{diag}(0,1), J\left(G, C, W_{l}, W_{r}\right) \leq 1$ corresponds to $\|W /(1+C G)\|_{\infty} \leq 1$. The performance filters $W_{l}(z)$ and $W_{r}(z)$ are chosen in such a way that they reflect the performance specifications that we want to achieve with the true system. Thus, a controller $C$ will be deemed satisfactory for the system $G_{0}$ if $\left[C G_{0}\right]$ is stable and if $J\left(G_{0}, C, W_{l}, W_{r}\right) \leq 1$.

As mentioned in the introduction, we want to design the new controller $\hat{C}(z)$ using an identified model $\hat{G}(z)=$ $G\left(z, \hat{\theta}_{N}\right)$ of $G_{0}(z)$. For this purpose, we will use a preselected nominal control design method:

Assumption 1 We have pre-selected a fixed nominal control design method which will map the identified model $\hat{G}$ to one controller $\hat{C}$. This control design method has been chosen in such a way that the controller $\hat{C}$ stabilizes $\hat{G}$ and achieves with this model a nominal performance level:

$J\left(\hat{G}, \widehat{C}, W_{l}, W_{r}\right) \leq \gamma<1$

where $\gamma$ is a fixed scalar.

One possible way to choose a control design method satisfying Assumption 1 is to choose for $\hat{C}$ the central controller of the four-block $H_{\infty}$ control design method with performance objective (10). When this controller $\hat{C}$ is applied to the true system $G_{0}$, the achieved performance will generically be poorer than the designed performance. However, by choosing the design criterion (10) with $\gamma<1$, we ensure that there is a whole set of systems $G$ around $\hat{G}$ that are also stabilized by $\hat{C}$ and that achieve $J\left(G, \hat{C}, W_{l}, W_{r}\right) \leq 1$. In the sequel, $\mathcal{D}_{a d m}\left(\hat{\theta}_{N}\right)$ denote the set containing all systems $G(z)$ having these properties. This set $\mathcal{D}_{a d m}\left(\hat{\theta}_{N}\right)$ of course contains $G\left(z, \hat{\theta}_{N}\right)$.

\section{Identification for control at the lowest cost}

Let us now proceed to our experiment design problem. As stated earlier, besides the standard requirement of an experiment which is informative enough, our objective is to determine the experimental conditions (i.e. $N$ and $\Phi_{r}(\omega)$ ) of the identification experiment on the loop $\left[C_{i d} G_{0}\right]$ in such a way that the model $\hat{G}$, identified through this experiment, delivers a controller $\hat{C}$ which stabilizes the unknown $G_{0}$ and achieves $J\left(G_{0}, \hat{C}, W_{l}, W_{r}\right) \leq 1$. Since $G_{0}$ is unknown but lies (with probability $\beta$ ) in the uncertainty region $\mathcal{D}\left(\hat{\theta}_{N}, P_{\theta}\right)$, this performance constraint will be replaced by the following checkable constraint.

Constraint 1 The experimental conditions (i.e. $N$ and $\left.\Phi_{r}(\omega)\right)$ of the identification experiment on the loop $\left[C_{i d} G_{0}\right]$ (see Section 2) must be such that the identified model $\hat{G}=G\left(z, \hat{\theta}_{N}\right)$ and the identified uncertainty region $\mathcal{D}\left(\hat{\theta}_{N}, P_{\theta}\right)$ have the property that $J\left(G, \hat{C}, W_{l}, W_{r}\right) \leq 1$ for all $G(z) \in \mathcal{D}\left(\hat{\theta}_{N}, P_{\theta}\right)$. In the previous expression, $\hat{C}$ is the controller designed from $\hat{G}$ using the control design method presented in Assumption 1.

Since $J\left(G, \hat{C}, W_{l}, W_{r}\right) \leq 1$ for all $G(z)$ in the set $\mathcal{D}_{a d m}\left(\hat{\theta}_{N}\right)$ defined in the last paragraph of Section 3 , Constraint 1 is ensured when $\mathcal{D}\left(\hat{\theta}_{N}, P_{\theta}\right)$ is a subset of this set $\mathcal{D}_{a d m}\left(\hat{\theta}_{N}\right)$. Since $P_{\theta}>0, \mathcal{D}\left(\hat{\theta}_{N}, P_{\theta}\right) \subseteq \mathcal{D}_{a d m}\left(\hat{\theta}_{N}\right)$ is always achievable if $N$ and/or $\Phi_{r}(\omega)$ are chosen large enough: see (6). Moreover, we have the following tradeoffs: the larger $N$ is chosen, the smaller $\Phi_{r}(\omega)$ can be while still verifying Constraint 1 . Conversely, the larger $\Phi_{r}(\omega)$ is chosen, the smaller $N$ can be while still verifying this constraint.

Based on the reasoning above, it is clear that many possible choices of experimental conditions allow one to fulfill Constraint 1. Among these, we seek to determine those inducing the smallest possible economical cost. We now define this cost. In normal operation the signals $u(t)$ (control signal) and $y(t)$ (the controlled variable which is in many cases the product) are given by: $y(t)=S_{i d} v(t)$ and $u(t)=-C_{i d} S_{i d} v(t)$. By applying an external signal $r(t)$ to the loop during the identification, we introduce disturbances $y_{r}(t)$ and $u_{r}(t)$ on top of the normal operation signals: see (2)-(3). Those disturbances induce a loss of production quality and thus entail an economical cost. Consequently, as far as the cost of the identification is concerned, the ideal closed-loop identification experiment would be, in many cases, one in which the normal operation signals $u(t)$ and $y(t)$ are used for a certain length $N$ without any external excitation, i.e. with $r(t)=0$. We show in Section 5 that such costless identification experiment can, in certain circumstances, fulfill Constraint 1. We also show how to compute the minimum number $N_{\text {min }}$ of measurements that are necessary to reach this objective.

In the cases where Constraint 1 can not be achieved with $r(t)=0$, the application of a nonzero external signal $r(t)$ for a certain amount of time is unavoidable, but we show how $N$ and $\Phi_{r}(\omega)$ can be chosen in order to 
achieve Constraint 1 with minimal economical cost. This economical cost will generally be a function of either the experiment time $N$, the power of the perturbations $y_{r}$ and $u_{r}$, or a combination of both. In the sequel, we will therefore distinguish three different situations and determine for each of them how we can optimally choose the experimental conditions.

Situation 1. The cost of the identification is mainly determined by the power of the perturbations $y_{r}(t)$ and $u_{r}(t)$. Based on the trade-off presented below Constraint 1, the experiment time $N$ is in this case chosen as large as we are allowed to. For such fixed $N$, the optimal power spectrum $\Phi_{r}(\omega)$ can subsequently be determined by minimizing the following cost function $\mathcal{J}_{r}$ :

$$
\begin{aligned}
\mathcal{J}_{r} & \left.=\alpha_{y}\left(\frac{1}{2 \pi} \int_{-\pi}^{\pi} \Phi_{y_{r}}(\omega) d \omega\right)+\alpha_{u}\left(\frac{1}{2 \pi} \int_{-\pi}^{\pi} \Phi_{u_{r}}(\omega) d \omega\right) 11\right) \\
& =\frac{1}{2 \pi} \int_{-\pi}^{\pi}\left(\alpha_{y}\left|G_{0}\left(e^{j \omega}\right) S_{i d}\left(e^{j \omega}\right)\right|^{2}+\alpha_{u}\left|S_{i d}\left(e^{j \omega}\right)\right|^{2}\right) \Phi_{r}(\omega) d \omega
\end{aligned}
$$

while guaranteeing Constraint 1. Here $\alpha_{y}$ and $\alpha_{u}$ are arbitrarily chosen scalars and $\Phi_{y_{r}}(\omega)$ and $\Phi_{u_{r}}(\omega)$ are the power spectra of the disturbance signals $y_{r}(t)$ and $u_{r}(t)$, respectively.

Situation 2. Situation 2 is the converse situation: the cost of the identification is mainly determined by the duration of the identification. Based on the trade-off presented below Constraint 1, the power spectrum $\Phi_{r}(\omega)$ of the to-be-applied signal $r(t)$ is in this case chosen at each frequency as large as the constraints on the actuators allow. For such fixed $\Phi_{r}(\omega)$, the optimal experiment time is the smallest experiment time $N_{\min }$ satisfying Constraint 1 . Note that, in this situation, the identification with $r=0$ is generally not the optimal experiment.

Situation 3. Situation 3 is where $N$ and $\Phi_{r}(\omega)$ are both important in the cost of the identification. In this situation, we can determine the optimal spectrum $\Phi_{r}(\omega)$ as in Situation 2 for different values of the length $N$. Since, for increasing values of $N$, the optimal cost function $\mathcal{J}_{r}$ decreases, such approach allows one to find the "optimal" combination for the duration of the identification and the induced disturbance $\mathcal{J}_{r}$.

\section{Costless identification experiment}

In this section, we show that there are conditions under which Constraint 1 can be achieved using an identification experiment on the closed loop $\left[C_{i d} G_{0}\right]$ without any external excitation signal $r(t)$, i.e. using only the excitation due to the noise $v(t)$. We have called such experiments "costless identification" since $r=0$ also implies
$\mathcal{J}_{r}=0$. The main condition for this to be possible is that Lemma 1 holds in this situation or, in other words, that the closed-loop identification experiment with $r(t)=0$ is informative enough i.e. that the expected identification criterion $\bar{V}(\theta)$ defined in (4) has a unique global minimum at $\theta_{0}$. A necessary and sufficient condition for this to hold is that $\epsilon(t, \theta)=\epsilon\left(t, \theta_{0}\right) \Longrightarrow \theta=\theta_{0}$. In closedloop identification with $r=0$, this condition specializes to the following result [21][page 392]: $\bar{V}(\theta)$ has a unique global minimum at $\theta_{0}$ if and only if, for any $\theta$,

$$
\begin{gathered}
H^{-1}(z, \theta)\left(1+C_{i d} G(z, \theta)\right)=H^{-1}\left(z, \theta_{0}\right)\left(1+C_{i d} G\left(z, \theta_{0}\right)\right) \\
\Longrightarrow \theta=\theta_{0} .
\end{gathered}
$$

A sufficient condition for this identifiability condition to hold, in the case of a linear time-invariant regulator $C_{i d}$ considered here, is that the regulator be sufficiently complex (i.e. of sufficiently high order). One can make this statement more precise by considering specific model structures. This has been done in [21][page 416] in the case where the model structure $\mathcal{M}$ is ARMAX or ARX. Here we extend the results of [21] to OE and BJ model structures. The model structures we consider are all special cases of the general class $\mathcal{M}=\{G(z, \theta) ; H(z, \theta)\}$ of model structures having the following parametrization

$G(z, \theta)=\frac{z^{-n_{k}} B(z, \theta)}{F(z, \theta) A(z, \theta)} H(z, \theta)=\frac{C(z, \theta)}{D(z, \theta) A(z, \theta)}$

with $A(z, \theta), B(z, \theta), C(z, \theta), D(z, \theta)$ and $F(z, \theta)$ polynomials of degree $n_{a}, n_{b}, n_{c}, n_{d}$ and $n_{f}$, respectively. We also consider that the controller $C_{i d}$ is represented as $C_{i d}=X / Y$ where $X$ and $Y$ are polynomials of degree $n_{x}$ and $n_{y}$, respectively.

We make the following assumption concerning the true closed-loop system and the parametric model structures.

Assumption 2 Consider the true system $\left[G\left(z, \theta_{0}\right) H\left(z, \theta_{0}\right)\right]$ in closed loop with the controller $C_{i d}=X / Y$ where $X$ and $Y$ are polynomials of degree $n_{x}$ and $n_{y}$, respectively. The identification is performed with a model structure that is contained in the general class of structures described above, with the following assumptions:

(1) for the true system (i.e. with $\theta=\theta_{0}$ ) there is no common factor in the polynomials $\{A, B, C\}$, the polynomials $\{B, F\}$ are coprime, and the polynomials $\{C, D\}$ are coprime;

(2) the controller polynomials $X$ and $Y$ are coprime;

(3) the closed loop denominator polynomial does not cancel any root of $C\left(z, \theta_{0}\right)$;

(4) the degrees $n_{k}, n_{a}, n_{b}, n_{c}, n_{d}, n_{f}$ used in the model structures are those of the true system (i.e. they are assumed known).

The coprimeness assumptions for the true system and for the controller parametrization are very weak. The 
assumption that the zeros of the noise model are not cancelled by the closed loop poles is generically satisfied given that the controller $C_{i d}$ is fixed, while the noise model numerator $C\left(z, \theta_{0}\right)$ is unknown. The only really restrictive assumption is the last one on exact knowledge of the degrees of the true system.

Proposition 1 Consider the closed loop identification described in Section 2 with $C_{i d}$ a linear time-invariant controller, and assume that $r(t)=0 \forall t$. Consider that the controller and the chosen model structure obey Assumption 2. Then, $\bar{V}(\theta)$ has a unique minimum in $\theta_{0}$ :

- in the case $\mathcal{M}=A R X(C=D=F=1)$ or $A R M A X$ $(D=F=1)$ if and only if either $n_{x}+n_{k}>n_{a}$ or $n_{y}>n_{b}$

- in the case $\mathcal{M}=B J(A=1)$ if and only if $n_{x}+n_{k}>$ $n_{d}+n_{f}$ or $n_{y}>n_{b}+n_{d}$

- in the case $\mathcal{M}=O E$ if and only if the controller $C_{i d}(z)$ is not identically zero.

Proof. The proof consists of verifying (12) for each of these model structures. For the ARX and ARMAX model structures, the result has been established in [21][page 416]. In the Box-Jenkins case, the left-hand side of (12) becomes:

$\frac{D(\theta)\left[F(\theta) Y+z^{-n_{k}} X B(\theta)\right]}{C(\theta) F(\theta)}=\frac{D\left(\theta_{0}\right)\left[F\left(\theta_{0}\right) Y+z^{-n_{k}} X B\left(\theta_{0}\right)\right]}{C\left(\theta_{0}\right) F\left(\theta_{0}\right)}$

The polynomials $C\left(\theta_{0}\right)$ and $D\left(\theta_{0}\right)$ are coprime by condition 1 of Assumption 2, while the polynomials $C\left(\theta_{0}\right)$ and $F\left(\theta_{0}\right) Y+z^{-n_{k}} X B\left(\theta_{0}\right)$ are coprime by condition 3 . Suppose now that $F\left(\theta_{0}\right)$ and $D\left(\theta_{0}\right)$ have a common polynomial factor $H$, i.e.

$F\left(\theta_{0}\right)=\bar{F}_{0} H, \quad D\left(\theta_{0}\right)=\bar{D}_{0} H$.

Since, by condition 4 of Assumption 2, $\operatorname{deg} C(\theta)+$ $\operatorname{deg} F(\theta)=\operatorname{deg} C\left(\theta_{0}\right)+\operatorname{deg} F\left(\theta_{0}\right)$, it follows that the solution set of $(13)$ is given by $C(\theta) F(\theta)=C\left(\theta_{0}\right) \bar{F}_{0} M$ and

$D(\theta)\left[F(\theta) Y+z^{-n_{k}} X B(\theta)\right]=\left[F\left(\theta_{0}\right) Y+z^{-n_{k}} X B\left(\theta_{0}\right)\right] \bar{D}_{0} M$

where, by condition 4 of Assumption 2, $M$ is an arbitrary polynomial of the same degree as that of $H$. Equivalently, the solution set is described by

$C(\theta) F(\theta)=C\left(\theta_{0}\right) \bar{F}_{0} M$

$D(\theta) F(\theta)=F\left(\theta_{0}\right) \bar{D}_{0} M+\xi z^{-n_{k}} X$

$D(\theta) B(\theta)=B\left(\theta_{0}\right) \bar{D}_{0} M-\xi Y$

where $\xi$ is an arbitrary polynomial. The solution set reduces to

$$
\begin{aligned}
& C(\theta) F(\theta)=C\left(\theta_{0}\right) \bar{F}_{0} M \\
& D(\theta) F(\theta)=F\left(\theta_{0}\right) \bar{D}_{0} M \\
& D(\theta) B(\theta)=B\left(\theta_{0}\right) \bar{D}_{0} M
\end{aligned}
$$

if and only if either $n_{x}+n_{k}>n_{d}+n_{f}$ or $n_{y}>n_{b}+n_{d}$. It now follows from the first and second of these equations, using (14), that

$$
\frac{C(\theta)}{D(\theta)}=\frac{C\left(\theta_{0}\right) \bar{F}_{0}}{\bar{D}_{0} F\left(\theta_{0}\right)}=\frac{C\left(\theta_{0}\right) \bar{F}_{0}}{\bar{D}_{0} H \bar{F}_{0}}=\frac{C\left(\theta_{0}\right)}{D\left(\theta_{0}\right)} .
$$

It follows from the second and third equation of (16) that

$$
\frac{B(\theta)}{F(\theta)}=\frac{B\left(\theta_{0}\right)}{F\left(\theta_{0}\right)} .
$$

From these last two expressions and condition 4 of Assumption 2 about the known degrees, we conclude that the only solution is $\theta=\theta_{0}$.

Finally, for the OE case, the left-hand side of (12) becomes $C_{i d} G(\theta)=C_{i d} G\left(\theta_{0}\right)$. As long as the linear timeinvariant controller $C_{i d}(z)$ is not identically zero, the latter implies $\theta=\theta_{0}$.

We have given conditions on the controller complexity under which an identification experiment with no external excitation delivers a cost function $\bar{V}(\theta)$ that has $\theta_{0}$ as its unique global minimum, i.e. the closed-loop identification with $r(t)=0$ is informative enough. It is then 13) trivial to show that, under those conditions, Constraint 1 can be made to hold.

Theorem 1 Consider a closed-loop identification experiment as presented in Section 2 with $r(t)=0$ and with $C_{i d}$ satisfying the conditions in Proposition 1. Then, Constraint 1 can always be achieved by using for the identification a set of (normal operation) input-output data of sufficient length $N$.

Proof. When $r=0$, expression (6) of $P_{\theta}^{-1}$ becomes $P_{\theta}^{-1}=N \mathcal{P}_{v}^{-1}\left(\theta_{0}\right)$. Since the experiment is informative enough, we have, via Lemma $1, \mathcal{P}_{v}^{-1}\left(\theta_{0}\right)>0$. Therefore, with $N$ sufficiently large, $P_{\theta}^{-1}=N \mathcal{P}_{v}^{-1}\left(\theta_{0}\right)$ can be made such that $\mathcal{D}\left(\hat{\theta}_{N}, P_{\theta}\right) \subseteq \mathcal{D}_{a d m}\left(\hat{\theta}_{N}\right)$ for any set $\mathcal{D}_{a d m}\left(\hat{\theta}_{N}\right)$ around $G\left(z, \hat{\theta}_{N}\right)$, which implies that Constraint 1 holds (see Section 4).

Theorem 1 shows that, if the controller complexity is chosen such that the closed-loop experiment with $r=0$ is informative enough, the identification leading to a new and satisfactory controller $\hat{C}$ for $G_{0}$ can be achieved without identification cost: we just need to measure the input and output signal in normal operation (i.e. with $r=0$ ) for a sufficient amount of time. The computation of the smallest data length $N_{\min }$ for which the robustness Constraint 1 is satisfied with $r=0$ is the special case of Situation 2 when the given reference excitation spectrum $\Phi_{r}(\omega)$ is equal to 0 ; a method to compute this minimal number of data will be presented in Section 6 . 


\section{Least costly identification when costless iden- tification is impossible}

An identification with $r=0$ may be impossible or non desirable for three reasons: i) the initial controller $C_{i d}$, which can often not be chosen by the user, is of lower complexity than is required by the conditions of Proposition 1, ii) the data length required to satisfy Constraint 1 (see Theorem 1) is unrealistic (e.g. too long) or iii) the controller $C_{i d}$ performs so badly that it is more economical to seek for the shortest identification duration (see Situation 2, Section 4). When identification with $r=0$ is ruled out, the least costly identification experiment for control is defined by the problems presented at the end of Section 4. Those problems involve the computation under Constraint 1 of either the power spectrum $\Phi_{r}(\omega)$ minimizing $\mathcal{J}_{r}$ for a given $N$ (Situations 1 and 3 ) or the smallest data length for a given $\Phi_{r}(\omega)$ (Situation 2).

We first examine Situation 1 in which the data length $N$ is fixed and one seeks to satisfy Constraint 1 with a power spetrum $\Phi_{r}(\omega)$ that minimizes the identification cost $\mathcal{J}_{r}$ defined in (11). We restrict our search to signals whose power spectrum $\Phi_{r}(\omega)$ can be written as [16]:

$\Phi_{r}(\omega)=R_{r}(0)+2 \sum_{i=1}^{m} R_{r}(i) \cos (i \omega) \geq 0 \quad \forall \omega$

where $m$ is a positive integer selected by the user. By designing a spectrum as (17), we always ensure that the corresponding identification experiment is informative enough and thus that Lemma 1 holds. Indeed, the parameters $R_{r}(i)(i=0 \ldots m)$ can be interpreted as the auto-correlation sequence of a signal that has been generated by a white noise passing through an FIR filter of length $m+1$.

Another important property of the parametrization (17) is that $P_{\theta}^{-1}$ and $\mathcal{J}_{r}$ (see (6) and (11)) are affine functions of the design variables $R_{r}(i)(i=0 \ldots m)$, as we show in the following proposition. Note that there are other parametrizations of $\Phi_{r}(\omega)$ that have the same property and could therefore also be considered here: e.g. $\Phi_{r}(\omega)=$ $\sum_{i=1}^{m} R_{r}(i) \delta\left(\omega-\omega_{i}\right)$ corresponding to a multisine signal $r(t)[7]$, or $\Phi_{r}(\omega)=\sum_{i=0}^{m} R_{r}(i)\left(\mathcal{B}_{i}\left(e^{j \omega}\right)+\mathcal{B}_{i}^{*}\left(e^{j \omega}\right)\right)$ where $\mathcal{B}_{i}\left(e^{j \omega}\right)$ are preselected basis functions [14].

Proposition 2 Consider the expression (6) of $P_{\theta}^{-1}$ and let $\Phi_{r}(\omega)$ be parametrized by (17). Let $\tilde{M}_{k}\left(\theta_{0}\right)$ be the sequence of Markov parameters of $F_{r} F_{r}^{*}$ i.e. $F_{r}\left(e^{j \omega}, \theta_{0}\right) F_{r}\left(e^{j \omega}, \theta_{0}\right)^{*}=\sum_{k=-\infty}^{\infty} \tilde{M}_{k}\left(\theta_{0}\right) e^{-j k \omega}$ with $F_{r}\left(z, \theta_{0}\right)$ as defined in (6). Then, $P_{\theta}^{-1} \in \mathbf{R}^{k \times k}$ can be written as:

$P_{\theta}^{-1}=\bar{M}\left(\theta_{0}\right)+\sum_{i=0}^{m} M_{i}\left(\theta_{0}, \sigma_{e}^{2}\right) R_{r}(i)$ where $\bar{M}\left(\theta_{0}\right)=N \mathcal{P}_{v}^{-1}\left(\theta_{0}\right), M_{0}\left(\theta_{0}, \sigma_{e}^{2}\right)=\frac{N}{\sigma_{e}^{2}} \tilde{M}_{0}\left(\theta_{0}\right)$, and $M_{i}\left(\theta_{0}, \sigma_{e}^{2}\right)=\frac{N}{\sigma_{e}^{2}}\left(\tilde{M}_{i}\left(\theta_{0}\right)+\tilde{M}_{i}^{T}\left(\theta_{0}\right)\right)$ for $i=1 \ldots m$. Moreover, the cost function $\mathcal{J}_{r}$ defined in (11) can be written as:

$$
\begin{aligned}
\mathcal{J}_{r}= & {\left[\alpha_{y} c_{0}\left(\theta_{0}\right)+\alpha_{u} d_{0}\left(\theta_{0}\right)\right] R_{r}(0) } \\
& +2 \sum_{i=1}^{m}\left[\alpha_{y} c_{i}\left(\theta_{0}\right)+\alpha_{u} d_{i}\left(\theta_{0}\right)\right] R_{r}(i),
\end{aligned}
$$

where the coefficients $c_{i}\left(\theta_{0}\right)$ and $d_{i}\left(\theta_{0}\right)$ are the Markov parameters of $G_{0} G_{0}^{*} S_{i d} S_{i d}^{*}$ and $S_{i d} S_{i d}^{*}$ respectively, i.e. $G_{0}\left(e^{j \omega}\right) G_{0}\left(e^{j \omega}\right)^{*} S_{i d}\left(e^{j \omega}\right) S_{i d}\left(e^{j \omega}\right)^{*}=\sum_{k=-\infty}^{\infty} c_{k}\left(\theta_{0}\right) e^{-j k \omega}$ and $S_{i d}\left(e^{j \omega}\right) S_{i d}\left(e^{j \omega}\right)^{*}=\sum_{k=-\infty}^{\infty} d_{k}\left(\theta_{0}\right) e^{-j k \omega}$.

Proof. The first part of the proposition is a direct consequence of Result 5.6 of [16] applied to the closed-loop expression for $P_{\theta}^{-1}$ as given in (6). The second part is a direct consequence of result 5.4 of [16] applied to $y_{r}(t)=G_{0} S_{i d} r(t)$ and $u_{r}(t)=S_{i d} r(t)$.

With the parametrization (17) for $\Phi_{r}(\omega)$, the experiment design problem corresponding to Situation 1 can then be formulated as follows.

Experiment Design Problem 1: Consider the closedloop identification experiment of Section 2 with a fixed number $N$ of data. Determine the parameters $R_{r}(i)(i=$ $0 \ldots m)$ of the spectrum $\Phi_{r}(\omega)$ in (17) which minimize $\mathcal{J}_{r}$, subject to satisfaction of Constraint 1.

We show in the sequel that this problem can be expressed as an LMI-based optimization problem [5]. For this purpose, we express the robust performance constraint $\bar{J}\left(\omega, G, \hat{C}, W_{l}, W_{r}\right) \leq 1 \forall G \in \mathcal{D}\left(\hat{\theta}_{N}, P_{\theta}\right)$ at one particular frequency $\omega$ as an LMI, linear in $P_{\theta}^{-1}$. Note that, according to (8), $J\left(G, \hat{C}, W_{l}, W_{r}\right) \leq 1 \forall G \in \mathcal{D}\left(\hat{\theta}_{N}, P_{\theta}\right)$ $\Longleftrightarrow \bar{J}\left(\omega, G, \hat{C}, W_{l}, W_{r}\right) \leq 1 \forall \omega$ and $\forall G \in \mathcal{D}\left(\hat{\theta}_{N}, P_{\theta}\right)$.

Proposition 3 Consider the controller $\hat{C}$ designed from the model $G\left(z, \hat{\theta}_{N}\right)$ using the control design method presented in Assumption 1. Consider the set $\mathcal{D}\left(\hat{\theta}_{N}, P_{\theta}\right)$ defined in (7). Then $\hat{C}$ achieves $\bar{J}\left(\omega, G, \hat{C}, W_{l}, W_{r}\right) \leq 1$ with all $G$ in $\mathcal{D}\left(\hat{\theta}_{N}, P_{\theta}\right)$ if and only if $\exists \tau(\omega)>0$, $\tau(\omega) \in \mathbf{R}$ and $\mathcal{L}(\omega)=-\mathcal{L}(\omega)^{T} \in \mathbf{R}^{(k+1) \times(k+1)}$ such that

$\tau(\omega) E\left(\omega, \hat{\theta}_{N}\right)-\mathcal{R}\left(\hat{\theta}_{N}\right)+j \mathcal{L}(\omega) \leq 0$

where $E\left(\omega, \hat{\theta}_{N}\right)=\Omega^{*}\left(e^{j \omega}\right)\left(\begin{array}{cc}I_{4} & 0 \\ 0 & -1\end{array}\right) \Omega\left(e^{j \omega}\right)$ with

$\Omega=\left(\begin{array}{c|c}\left(I_{2} \otimes\left(W_{r}\left(\begin{array}{c}\hat{C} \\ 1\end{array}\right)\right)\right) W_{l} & 0 \\ \hline 0 & 1\end{array}\right)\left(\begin{array}{cc}Z_{N} & 0 \\ Z_{D} & 1 \\ Z_{D}+\hat{C} Z_{N} & 1\end{array}\right)$ 
$\Omega(z)$ has the dimension $5 \times(k+1)$ and $\otimes$ denotes the Kronecker product. Finally:

$\mathcal{R}\left(\hat{\theta}_{N}\right)=\left(\begin{array}{c}I_{k} \\ -\hat{\theta}_{N}^{T}\end{array}\right) P_{\theta}^{-1}\left(\begin{array}{c}I_{k} \\ -\hat{\theta}_{N}^{T}\end{array}\right)^{T}+\left(\begin{array}{cc}0 & 0 \\ 0 & -\chi\end{array}\right) \in \mathbf{R}^{(k+1) \times(k+1)}$

Proof. See Appendix A.

The previous proposition shows that Constraint 1 can be replaced by LMI's at each frequency, linear in $P_{\theta}^{-1}$ and thus, via Proposition 2, linear in the decision variables $R_{r}(i)$ of Experiment Design Problem 1. Combining this fact with the fact that its cost function $\mathcal{J}_{r}$ is also linear in $R_{r}(i)$, Experiment Design Problem 1 would be solvable exactly if the parametrizations of $P_{\theta}^{-1}$ and $\mathcal{J}_{r}$ with respect to the design variables $R_{r}(i)$ were not functions of the unknown $\theta_{0}$ and $\sigma_{e}^{2}$, and if condition (18) was not a function of the to-be-identified $\hat{\theta}_{N}$. This difficulty can be circumvented by using a-priori estimates for those quantities: $\theta_{o, e s t}, \sigma_{e, e s t}^{2}$ and $\hat{\theta}_{N, e s t}$. The solution is then obtained by solving the LMI optimization problem described in Theorem 2 below. In Section 7 we shall present an attractive alternative to the reliance on a-priori estimates; it is based on a small adaptation of the LMI problem of Theorem 2.

Theorem 2 Consider the approximations $\theta_{0} \approx \theta_{o, e s t}$, $\hat{\theta}_{N} \approx \hat{\theta}_{N, \text { est }}$ and $\sigma_{e}^{2} \approx \sigma_{e, e s t}^{2}$ and the shorthand notations: $c_{i}=c_{i}\left(\theta_{o, e s t}\right), d_{i}=d_{i}\left(\theta_{o, e s t}\right), \bar{M}=\bar{M}\left(\theta_{o, e s t}\right)$, $M_{i}=M_{i}\left(\theta_{o, e s t}, \sigma_{e, e s t}^{2}\right)$ and $E(\omega)=E\left(\omega, \hat{\theta}_{N, \text { est }}\right)$. Then the auto-correlation sequence $R_{r}(i)(i=0 \ldots m)$ which solves Experiment Design Problem 1 is the solution of the following LMI optimization problem:

$\min _{R_{r}(i)(i=0 \ldots m)}\left[\alpha_{y} c_{0}+\alpha_{u} d_{0}\right] R_{r}(0)+2 \sum_{i=1}^{m}\left[\alpha_{y} c_{i}+\alpha_{u} d_{i}\right] R_{r}(i)$

under the constraint that there exist a matrix $Q=Q^{T}$ of appropriate dimension, $\tau(\omega)>0, \tau(\omega) \in \mathbf{R}$ and $\mathcal{L}(\omega)=$ $-\mathcal{L}(\omega)^{T} \in \mathbf{R}^{(k+1) \times(k+1)}$ such that

$\begin{aligned} \tau(\omega) E\left(e^{j \omega}\right)- & \left(\begin{array}{c}I_{k} \\ -\hat{\theta}_{N, e s t}^{T}\end{array}\right)\left(\bar{M}+\sum_{i=0}^{m} M_{i} R_{r}(i)\right)\left(\begin{array}{c}I_{k} \\ -\hat{\theta}_{N, \text { est }}^{T}\end{array}\right)^{T} \\ & -\left(\begin{array}{cc}0 & 0 \\ 0 & -\chi\end{array}\right)+j \mathcal{L}(\omega) \leq 0 \quad \forall \omega\end{aligned}$

and that $\left(\begin{array}{cc}Q-A^{T} Q A & C^{T}-A^{T} Q B \\ C-B^{T} Q A & D+D^{T}-B^{T} Q B\end{array}\right) \geq 0$ with the following definitions of $A, B, C, D$ :

$$
\begin{gathered}
A=\left(\begin{array}{cc}
0 & 0 \\
I_{m-1} & 0
\end{array}\right) \\
\left.R_{r}(1) R_{r}(2) \ldots R_{r}(m)\right)
\end{gathered} \quad B=\left(\begin{array}{llll}
1 & 0 & \ldots & 0
\end{array}\right)
$$

The optimal spectrum $\Phi_{r}(\omega)$ can then be computed using (17).

Proof. As shown in [16] via the Positive Real Lemma, the constraint that there exists a symmetric matrix $Q$ such that (20) holds is a necessary and sufficient condition for $R_{r}(0)+2 \sum_{i=1}^{m} R_{r}(i) \cos (i \omega)$ to be positive at each $\omega$ and thus for (17) to represent a spectrum. Consequently, the result in this theorem is a direct consequence of Propositions 2 and 3.

Comment 1. Condition (19) must be considered at every frequency. This is impossible in practice. The optimal spectrum can nevertheless be approximated by using a finite frequency grid. An exact but more cumbersome solution consists of using the Kalman-Yakubovitch-Popov (KYP) lemma [19]: see Appendix B.

Comment 2. Additional constraints such as $\Phi_{u}(\omega)<$ $\beta_{u}(\omega)$ and $\Phi_{y}(\omega)<\beta_{y}(\omega) \forall \omega$ can also be treated (see $[16])$.

We now consider the experiment design problem corresponding to Situation 2.

Experiment Design Problem 2. Consider the closedloop identification experiment of Section 2. Consider also that the power spectrum $\Phi_{r}(\omega)$ of the excitation signal $r(t)$ is given and leads to an informative enough identification experiment. Determine then the smallest length $N$ of a part of $r(t)$ that must be applied to $\left[C_{i d} G_{0}\right]$ in order to fulfill Constraint 1.

Since (6) shows that $P_{\theta}^{-1}$ is linear in the decision variable $N$ of Experiment Design Problem 2, it can be solved in a similar way and with the same approximations as Experiment Design Problem 1.

Theorem 3 Consider the approximations $\theta_{0} \approx \theta_{o, \text { est }}$, $\hat{\theta}_{N} \approx \hat{\theta}_{N, \text { est }}$ and $\sigma_{e}^{2} \approx \sigma_{e, e s t}^{2}$ and the shorthand notations: $\mathcal{P}_{r}^{-1}\left(\Phi_{r}(\omega)\right)=\mathcal{P}_{r}^{-1}\left(\Phi_{r}(\omega), \theta_{o, e s t}, \sigma_{e, e s t}^{2}\right), \mathcal{P}_{v}^{-1}=$ $\mathcal{P}_{v}^{-1}\left(\theta_{o, \text { est }}\right)(\operatorname{see}(6))$ and $E(\omega)=E\left(\omega, \hat{\theta}_{N, \text { est }}\right)($ see $(18))$. Then, the minimum duration $N$ which solves Experiment Design Problem 2 is the solution (rounded up to the nearest integer) of the LMI optimization problem minimizing $N$ under the constraint that there exist $\tau(\omega)>0$, $\tau(\omega) \in \mathbf{R}$ and $\mathcal{L}(\omega)=-\mathcal{L}(\omega)^{T} \in \mathbf{R}^{(k+1) \times(k+1)}$ such that the constraint (19) with $\bar{M}+\sum_{i=0}^{m} M_{i} R_{r}(i)$ replaced by $N\left(\mathcal{P}_{r}^{-1}\left(\Phi_{r}(\omega)\right)+\mathcal{P}_{v}^{-1}\right)$ holds at each frequency $\omega$. 


\section{A robust optimal design procedure}

Theorems 2 and 3 require that initial estimates of $\theta_{0}, \hat{\theta}_{N}$ and $\sigma_{e}^{2}$ be used. If those approximations are not accurate, this could lead to poor results. In this section, we present a procedure that renders the computed optimal design solution more robust w.r.t. these unknown quantities. Very often, estimates of $\theta_{0}$ and $\sigma_{e}^{2}$ are available, because the initial controller $C_{i d}$ has been computed from an identified model. This initial identification delivers estimates not only of $\theta_{0}$ and $\sigma_{e}^{2}$, but also of uncertainty regions for those quantities. It is then possible to deduce from this initial identification a (truncated) Gaussian probability density function which defines the likelihood of each element of these uncertainty regions. If this is not possible, the density functions are chosen uniform over the uncertainty regions. The estimate, the uncertainty region and the probability density function of the to-be-identified $\hat{\theta}_{N}$ are typically chosen equal to those of $\theta_{0}$. To summarize, from an initial identification one can assume that $q_{0}=\left(\begin{array}{lll}\theta_{0}^{T} \hat{\theta}_{N}^{T} & \sigma_{e}^{2}\end{array}\right)^{T}$ lies in a set $\mathcal{Q}$ and that the likelihood of the event $q=q_{0}$ is given by a probability function $p(q)$. Based on this information, one can robustify the procedure that consists in adopting a unique and possibly poor estimate of $q_{0}$ for the design of the experimental conditions. This is achieved by the use of randomized algorithms (see e.g. [23,22]). In the case of Experiment Design Problem 2, we want to determine the smallest duration $N$ for which Constraint 1 is verified for all possible values of $q_{0}$ in $\mathcal{Q}$. This is equivalent to computing an estimate $\hat{N}$ of $\sup _{q \in \mathcal{Q}} N_{q}$ where $N_{q}$ is the solution obtained by Theorem 3 with the approximation $q$. Considering $N_{q}$ as a function of $q$, this can be done [22] with accuracy $\epsilon$ and confidence ${ }^{1} \delta$ by generating $n \geq \ln \left(\delta^{-1}\right) / \ln \left((1-\epsilon)^{-1}\right)$ estimates $q_{j}$ of $q_{0}$ according to the probability density function $p(q)$, and by determining the corresponding optimal $N_{q_{j}}$ for each of these estimates $q_{j}$ using Theorem 3 . An estimate $\hat{N}$ of $\sup _{q \in \mathcal{Q}} N_{q}$ is then given by $\sup _{q_{j}(j=1 \ldots n)} N_{q_{j}}$. In the case of Experiment Design Problem 1, the approach above can not be considered since we determine the parameters $R_{r}(i)(i=0 \ldots m)$ of $\Phi_{r}(\omega)$ rather than $\Phi_{r}(\omega)$ itself. Instead, the so-called scenario-approach can be considered (see e.g. [23, Chapter 12]). This approach is also based on a randomized algorithm which uses the probability density function $p(q)$. However, whereas for Experiment Design Problem 2 we apply Theorem 3 a fixed number of times for estimates of $q_{0}$ randomly generated from $p(q)$, for Experiment Design Problem 1 we solve the optimization problem only once, but with several robust performance constraints (19), each evaluated at a different estimate of $q_{0}$ randomly generated from $p(q)$. The scenario approach is thus similar to the idea presented in [14] (and [3]) which was based on a gridding of $\mathcal{Q}$.

\footnotetext{
1 This means that $\operatorname{Pr}\left(\operatorname{Pr}\left(N_{q}>\hat{N}\right) \leq \epsilon\right) \geq 1-\delta$.
}

\section{Simulation results}

In order to illustrate our results, we consider as true system the following ARX system [15]: $y(t)=$ $\left(z^{-3} B_{0}(z)\right) /\left(A_{0}(z)\right) u(t)+(1) /\left(A_{0}(z)\right) e(t)$ with $B_{0}(z)=$ $0.10276+0.18123 z^{-1}, A_{0}(z)=1-1.99185 z^{-1}+$ $2.20265 z^{-2}-1.84083 z^{-3}+0.89413 z^{-4}$, and $e(t)$ a realization of a white noise process of variance $\sigma_{e}^{2}=0.5$. The control performance criterion $J\left(G, C, W_{l}, W_{r}\right)$ focuses on the sensitivity function. It is defined as in (8) with the filters: $W_{l}(z)=\operatorname{diag}(0, W(z)), W_{r}(z)=\operatorname{diag}(0,1)$ with $W(z)=\left(0.5165-0.4632 z^{-1}\right) /\left(1-0.999455 z^{-1}\right)$.

The true system initially operates in closed loop with a controller $C_{i d}$ which has been designed using an initial estimate of the true system $\theta_{o \text {,est }}=$ $(-1.9755,2.1965,-1.8495,0.8881,0.0817,0.172)^{T}$ and the 4-block $H_{\infty}$ control design method of [8] that satisfies Assumption 1: $C_{i d}=\left(0.036249(z+0.9244)\left(z^{2}-\right.\right.$ $1.951 z+1.101)\left(z^{2}-0.5109 z+0.8248\right)\left(z^{2}-0.1828 z+\right.$ $0.9416)) /\left((z-0.9995)\left(z^{2}-1.002 z+0.3641\right)\left(z^{2}-\right.\right.$ $\left.1.279 z+0.835)\left(z^{2}-0.1746 z+0.9229\right)\right)$

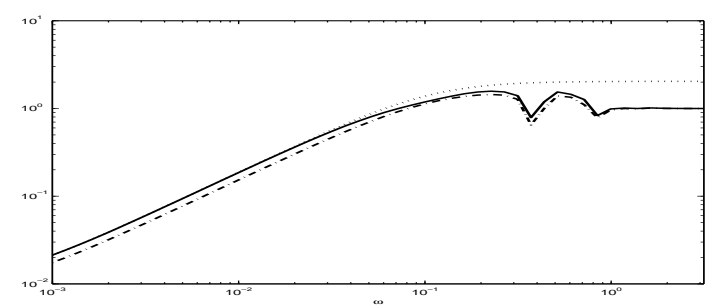

Fig. 2. Costless identification with $N=4901$ : $\sup _{G \in \mathcal{D}\left(\hat{\theta}_{N}, P_{\theta}\right)}\left|\frac{1}{1+\hat{C}\left(e^{j \omega}\right) G\left(e^{j \omega}\right)}\right|$ (solid), $\left|(1+\hat{C} \hat{G})^{-1}\right|$ (dashdot) and $|W|^{-1}$ (dotted)

Costless identification. The order of the initial controller $C_{i d}$ is sufficient for the experiment with $r=0$ to be informative enough (see Proposition 1). Thus, Constraint 1 can be verified with an identification experiment using normal operation signals $y$ and $u$. The minimal length for this data set can then be determined using Theorem 3 with $\Phi_{r}(\omega)=0 \forall \omega$. This theorem is applied here using the initial estimates $\theta_{0} \approx \theta_{o, \text { est }}$ and $\hat{\theta}_{N} \approx \theta_{o, e s t}$ (an estimate of $\sigma_{e}^{2}$ is not necessary since $\mathcal{P}_{v}^{-1}\left(\theta_{0}\right)$ is not a function of $\left.\sigma_{e}^{2}\right)$. This delivers a minimal length $N_{\min }=4901$.

In order to verify the validity of this result, we have measured 4901 samples of the signals $y(t)$ and $u(t)$ obtained in normal operation on the loop $\left[C_{i d} G_{0}\right]$ and we have identified a model $\hat{G}=G\left(z, \hat{\theta}_{N}\right)$ along with its uncertainty region $\mathcal{D}\left(\hat{\theta}_{N}, P_{\theta}\right)$. From $\hat{G}$, we have then designed a controller $\hat{C}$ using the method of [8] and we have verified whether $\hat{C}$ achieves $J\left(G, \hat{C}, W_{l}, W_{r}\right) \leq 1$ with all $G$ in $\mathcal{D}\left(\hat{\theta}_{N}, P_{\theta}\right)$, or equivalently $\left|\frac{1}{1+\hat{C}\left(e^{j \omega}\right) G\left(e^{j \omega}\right)}\right| \leq$ $\left|W\left(e^{j \omega}\right)\right|^{-1}$ for all $G$ in $\mathcal{D}\left(\hat{\theta}_{N}, P_{\theta}\right)$. This is indeed the 
case as can be seen in Figure 2. Moreover, we also observe in Figure 2 that $\sup _{G \in \mathcal{D}\left(\hat{\theta}_{N}, P_{\theta}\right)}\left|\frac{1}{1+\hat{C}\left(e^{j \omega}\right) G\left(e^{j \omega}\right)}\right|=$ $\left|W\left(e^{j \omega}\right)\right|^{-1}$ in low frequencies. Consequently, $N=4901$ is indeed the smallest $N$ for which, in this example, Constraint 1 holds with $\Phi_{r}(\omega)=0 \forall \omega$.

Sensitivity to the initial estimates. The initial estimate $\theta_{o, e s t}$ chosen to approximate the unknown quantities $\theta_{0}$ and $\hat{\theta}_{N}$ has, in this example, delivered accurate results, as shown by Figure 2. This may not always be the case. Thus, it is always safer to compute the minimal data length $N$ using the method proposed in Section 7. We illustrate the application of this method to our example. In order to generate multiple estimates of $\theta_{0}$ (which are then used to approximate both the true $\theta_{0}$ and $\hat{\theta}_{N}$ ), we have used the information delivered by the initial identification which had delivered $\theta_{o, e s t}$. This was an open-loop identification with ${ }^{2} \Phi_{u}(\omega)=1 \forall \omega$ and $N=500$. Using the covariance matrix of $\theta_{o, e s t}$, we have randomly generated 46 parameter vectors $\theta_{i}(i=1 \ldots 46)$ around $\theta_{o, e s t} ; 46$ samples correspond to an accuracy and a confidence of $80 \%$. For each of these estimates, we have applied Theorem 3 and we have thus obtained 46 different lengths $N_{\theta_{i}}$. A more robust choice of the length $N$ is then (see Section 7): $\max _{\theta_{i}} N_{\theta_{i}}=5897$. Note that the standard deviation of the 46 found values of $N_{\theta_{i}}$ was 383 .

Least costly identification with external excitation. For the same example, we have also studied the effect of applying least costly identification signals for a range of data lengths that were too short to lead to identifiability using only the noise excitation. Thus, we have computed the optimal signal spectra $\Phi_{r}(\omega)$ using Theorem 2 for data lenghts $N$ ranging from 500 to 4,500 by steps of 500 . For each of these data lenghts, we have then compared the identification cost $\mathcal{J}_{r}$ resulting from the application of the optimal excitation signal (when $m=10$ in (17)) with the cost that would result by applying a white noise reference excitation (i.e. $m=0$ in (17)) with a variance that is sufficient to satisfy the robust performance Constraint 1. The comparison between the cost of the least costly experiment and the cost of a corresponding identification experiment with white noise excitation is shown in Figure 3. As can be seen from this figure, the use of an optimally designed excitation signal $r$ reduces the identification cost by a factor of 2 to 3 whatever the data length. Note that similar comparisons leading to similar conclusions can be found for the open-loop identification case in [13].

\section{Conclusions}

We have proposed a new paradigm for optimal experiment design in an identification for robust control con-

\footnotetext{
2 This initial identification was too cheap to verify Constraint 1.
}

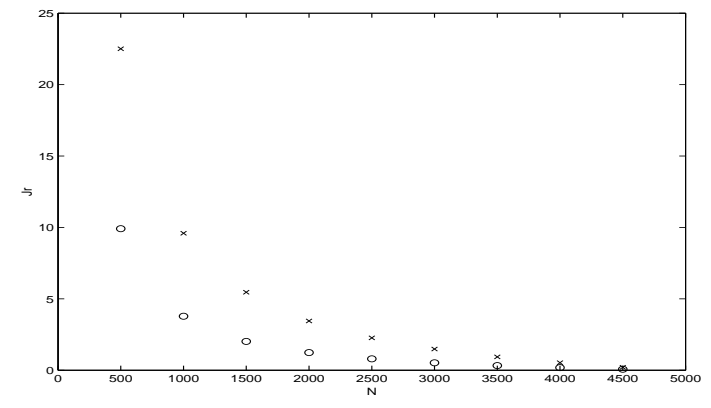

Fig. 3. Cost of the least costly experiment that satisfies Constraint 1 (circles) and of the white noise experiment that satisfies the same constraint (crosses), for different values of the data length.

text, where the objective is to design an identification experiment at the smallest possible cost that just meets the demands of the robust control performance in terms of the quality constraints on the estimated model. In other words, the identification cost must be as small as possible while still delivering a model uncertainty set that just meets the robust control performance constraints. The cost is expressed either as the experiment time, or in terms of a measure of the deterioration of the closedloop performance, during the identification experiment.

Even though these are classical assumptions for optimal experimental design in the PE framework, the assumptions that the true system can be described by the chosen model structure $\mathcal{M}$ and the use of the covariance matrix expression asymptotic in the data length are the restrictive aspects of the methodology. The first assumption can be relaxed. In the recent contribution [2], the "least costly identification for control" framework is indeed extended to the case of model structures $\mathcal{M}$ of reduced order. Further research will also focus on the relaxation of the second assumption. See e.g. the results in [13][Chapter 4] and [2]. Note that the counterpart for both relaxations is that only model structures linear in the parameter vector can be considered.

\section{References}

[1] X. Bombois, M. Gevers, G. Scorletti, and B.D.O. Anderson. Robustness analysis tools for an uncertainty set obtained by prediction error identification. Automatica, 37(10):16291636, October 2001.

[2] X. Bombois and M. Gilson. Cheapest identification experiment with guaranteed accuracy in the presence of undermodeling. In 14th IFAC Symposium on System Identification, pages 505-510, Newcastle, 2006.

[3] X. Bombois, G. Scorletti, M. Gevers, R. Hildebrand, and P.M.J. Van den Hof. Cheapest open-loop identification for control. In CD-ROM Proc. 33rd IEEE Conf on Decision and Control, pages 382-387, The Bahamas, December 2004.

[4] X. Bombois, G. Scorletti, P.M.J. Van den Hof, and M. Gevers. Least costly identification experiment for control: a solution based on a high-order model approximation. In $C D-R O M$ 
Proc. American Control Conference, Boston, MA, USA, July 2004.

[5] S. Boyd, L. El Ghaoui, E. Feron, and V. Balakrishnan. Linear Matrix Inequalities in System and Control Theory. SIAM Studies in Applied Mathematics, Philadelphia, 1994.

[6] B. Cooley and J. Lee. Control-relevant experiment design for multivariable systems described by expansions in othonormal bases. Automatica, 37(2):273-281, February 2001.

[7] E. Van den Eijnde and J. Schoukens. On the design of optimal excitation signals. In Proc. 9th IFAC/IFORS Symp. on Identification and System Parameter Estimation, pages 827-832, Budapest, Hungary, 1991.

[8] G. Ferreres and V. Fromion. $H_{\infty}$ control for a flexible transmission system. In CD-ROM Proc. European Control Conference, Brussels, Belgium, 1997.

[9] U. Forssell and L. Ljung. Some results on optimal experiment design. Automatica, 36:749-756, 2000.

[10] M. Gevers and L. Ljung. Optimal experiment designs with respect to the intended model application. Automatica, 22:543-554, 1986.

[11] R. Hildebrand and M. Gevers. Identification for control: optimal input design with respect to a worst-case $\nu$-gap cost function. SIAM Journal on Control and Optimization, 41(5):1586-1608, March 2003.

[12] H. Hjalmarsson, M. Gevers, and F. De Bruyne. For modelbased control design, closed-loop identification gives better performance. Automatica, 32:1659-1673, 1996.

[13] H. Jansson. Experiment design with applications in Identification for Control. $\mathrm{PhD}$ thesis, Royal Institute of Technology, Stockholm, Sweden, 2004.

[14] H. Jansson and H. Hjalmarsson. Mixed $H_{\infty}$ and $H_{2}$ input design for identification. In CD-ROM Proc. 43 rd IEEE Conf on Decision and Control, pages 388-393, The Bahamas, 2004.

[15] I.D. Landau, D. Rey, A. Karimi, A. Voda, and A. Franco. A flexible transmission system as a benchmark for robust digital control. European Journal of Control, 1(2):77-96, 1995.

[16] K. Lindqvist. On experiment design in identification of smooth linear systems. Licenciate Thesis, Royal Institute of Technology, Stockholm, Sweden, 2001.

[17] L. Ljung. System Identification: Theory for the User, 2nd Edition. Prentice-Hall, Englewood Cliffs, NJ, 1999.

[18] G. Meinsma, Y. Shrivastava, and M. Fu. A dual formulation of mixed $\mu$ and on the losslessness of $(D, G)$ scaling. IEEE Transactions on Automatic Control, 42(7):1032-1036, 1997.

[19] V.M. Popov. Hyperstability of Control Systems. SpringerVerlag, New-York, 1973.

[20] D.E. Rivera, H. Lee, M.W. Braun, and H.D. Mittelmann. Plant-friendly system identification: a challenge for the process industries. In Proc. 13th IFAC Symposium on System Identification, pages 917-922, Rotterdam, 2003.

[21] T. Söderström and P. Stoica. System Identification. PrenticeHall International, Hemel Hempstead, Hertfordshire, 1989.

[22] R. Tempo, E.W. Bai, and F. Dabbene. Probabilistic robustness analysis: explicit bound for the minimum number of samples. Systems and Control Letters, 30:237-242, 1997.

[23] R. Tempo, G. Calafiore, and F. Dabbene. Randomized algorithms for analysis and control of uncertain systems. Springer Verlag, New York, 2004.

\section{A Proof of Proposition 3}

Lemma 2 Let $A=A^{*} \in \mathcal{C}^{n \times n}$. Then

$y^{T} A y \leq 0, \quad$ for all $y \in \mathbf{R}^{n}$

if and only if

there exists $\mathcal{L}=-\mathcal{L}^{T} \in \mathbf{R}^{n \times n}$ such that $A+j \mathcal{L} \leq 0$. (A.2)

Proof of Lemma 2. (A.2) $\Rightarrow$ (A.1). Note that, for any $y \in \mathbf{R}^{n}$ and for any $\mathcal{L}=-\mathcal{L}^{T} \in \mathbf{R}^{n \times n}, y^{T} j \mathcal{L} y=0$. Condition (A.2) implies then that, for all $y \in \mathbf{R}^{n}, y^{T} A y \leq 0$.

(A.1) $\Rightarrow($ A.2) is proved by contradiction. Assume thus that (A.1) holds and that:

there is no $\mathcal{L}=-\mathcal{L}^{T} \in \mathbf{R}^{n \times n}$ such that $A+j \mathcal{L} \leq 0$. (A.3)

Since Condition (A.1) holds, we have that:

for all $S=S^{T} \geq 0 \in \mathbf{R}^{n \times n}, \quad \operatorname{Trace}(S A) \leq 0$.

On the other hand, based on Lemma III.1 in [18], condition (A.3) is equivalent to the fact that there exists $W=W^{*} \geq 0\left(W \in \mathcal{C}^{n \times n}\right)$ such that:

$\operatorname{Trace}(W(A+j \mathcal{L}))>0$, for all $\mathcal{L}=-\mathcal{L}^{T} \in \mathbf{R}^{n \times n}$

Note now that

$$
\operatorname{Trace}(W j \mathcal{L})=-\operatorname{Trace}(\operatorname{Im}(W) \mathcal{L})=2 \sum_{i=1}^{\frac{n(n-1)}{2}} w_{i} l_{i}
$$

where $w_{i}\left(i=1 \ldots \frac{n(n-1)}{2}\right)$ are the $\frac{n(n-1)}{2}$ non-zeros entries of the upper-triangular part of the skew symmetric matrix $\operatorname{Im}(W)$ and $l_{i}$ are the corresponding entries of $\mathcal{L}$ in the same location. Thus, (A.5) is equivalent to:

$$
\operatorname{Trace}(W A)+2 \sum_{i=1}^{\frac{n(n-1)}{2}} w_{i} l_{i}>0 \text { for all } l_{i} \in \mathbf{R}
$$

which is equivalent to $w_{i}=0$ (i.e. the matrix $W$ is real) and $\operatorname{Trace}(W A)>0$. The latter is in contradiction with (A.4) and the proof is thus completed.

Proof of Proposition 3. From [1], for $G \in \mathcal{D}\left(\hat{\theta}_{N}, P_{\theta}\right)$, $\bar{J}\left(\omega, G, \hat{C}, W_{l}, W_{r}\right) \leq 1$ can be written as:

$\mathcal{G}^{*}\left(e^{j \omega}\right)\left(\begin{array}{cc}x^{*} x I_{2} & 0 \\ 0 & -1\end{array}\right) \mathcal{G}\left(e^{j \omega}\right) \leq 0$ 
with $x=W_{r}\left(e^{j \omega}\right)\left(\hat{C}\left(e^{j \omega}\right) 1\right)^{T}$ and

$\mathcal{G}=\left(\begin{array}{cc}W_{l} & 0 \\ 0 & 1\end{array}\right)\left(\begin{array}{c}Z_{N} \theta \\ 1+Z_{D} \theta \\ 1+\left(Z_{D}+\hat{C} Z_{N}\right) \theta\end{array}\right)$

If we define $\bar{\theta}=\left(\theta^{T} 1\right)^{T}$, expression (A.6) can be rewritten as $\bar{\theta}^{T} E\left(\omega, \hat{\theta}_{N}\right) \bar{\theta} \leq 0$. On the other hand, the constraint $\theta \in U$ of (7) can be rewritten as $\bar{\theta}^{T} \mathcal{R}\left(\hat{\theta}_{N}\right) \bar{\theta} \leq$ 0 . Consequently, proving that $\bar{J}\left(\omega, G, \hat{C}, W_{l}, W_{r}\right) \leq 1$ for all $G \in \mathcal{D}\left(\hat{\theta}_{N}, P_{\theta}\right)$ is equivalent to proving that $\bar{\theta}^{T} E\left(\omega, \hat{\theta}_{N}\right) \bar{\theta} \leq 0$ for all $\bar{\theta} \in \mathbf{R}^{(k+1) \times(k+1)}$ such that $\bar{\theta}^{T} \mathcal{R}\left(\hat{\theta}_{N}\right) \bar{\theta} \leq 0$. Using the $\mathcal{S}$ procedure [5], the latter is equivalent to proving that $\exists \tau(\omega)>0$ such that, for all $\bar{\theta} \in \mathbf{R}^{(k+1) \times(k+1)}, \bar{\theta}^{T}\left(\tau(\omega) E\left(\omega, \hat{\theta}_{N}\right)-\mathcal{R}\left(\hat{\theta}_{N}\right)\right) \bar{\theta} \leq 0$. The proposition then follows by applying Lemma 2 .

\section{B Kalman Yakubovitch Popov (KYP) Lemma}

Theorems 2 and 3 show that the robust performance constraint $J\left(G, \hat{C}, W_{l}, W_{r}\right) \leq 1 \forall G \in \mathcal{D}\left(\hat{\theta}_{N}, P_{\theta}\right)$ can be treated by an infinite set of LMI's (i.e. the LMI condition (18) at each $\omega$ ). In this appendix, we show in Proposition 4 that we can replace this infinite set of LMI's by a single LMI by removing the frequency dependence of condition (18) using the KYP lemma. Before applying the KYP to this condition, note that condition (18) is not the unique way to express $\bar{J}\left(\omega, G, \hat{C}, W_{l}, W_{r}\right) \leq 1$ $\forall G \in \mathcal{D}\left(\hat{\theta}_{N}, P_{\theta}\right)$ as an LMI linear in $P_{\theta}^{-1}$ : in [1], we developed another LMI condition. That LMI condition involves the real part of a frequency-dependent matrix. Note also that, in [14], the authors apply the Real Positive (RP) lemma (a special case of the KYP lemma) to a LMI condition of the type in [1]. For this purpose, they need to multiply the LMI by the least common denominator of its entries. While entirely correct, this approach can lead to a final frequency-independent LMI which has an unnecessarily large state-space representation. Consequently, we have here developed the frequencydependent condition (18) in such a way that the corresponding frequency-independent LMI obtained via the KYP lemma has the lowest possible state-space representation. This frequency-independent LMI is given in the following proposition:

Proposition 4 Consider Proposition 3 and an arbitrary positive integer $b$. Define $\mathcal{B}(z)=\left(1, z^{-1}, \ldots, z^{-b}\right)^{T}$. Then, condition (18) holds for all $\omega$ (or equivalently $\left.J\left(G, \hat{C}, W_{l}, W_{r}\right) \leq 1 \forall G \in \mathcal{D}\left(\hat{\theta}_{N}, P_{\theta}\right)\right)$ if there exists $P_{f}=P_{f}^{T}$ and $P_{\lambda}=P_{\lambda}^{T}$ of appropriate dimensions, real numbers $\lambda_{i}, i=0 \ldots b$ and $L_{p} \in \mathbf{R}^{(k+1) \times(b+1)(k+1)}$ of the form:

$$
L_{p}=\left(\begin{array}{ccccc}
0 & l_{12}^{T} & l_{13}^{T} & \ldots & l_{1(k+1)}^{T} \\
-l_{12}^{T} & 0 & l_{23}^{T} & \ldots & l_{2(k+1)}^{T} \\
\ldots & \ldots & \ldots & \ldots & \ldots \\
-l_{1(k+1)}^{T} & -l_{2(k+1)}^{T} & -l_{3(k+1)}^{T} & \ldots & 0
\end{array}\right)
$$

with $l_{i j} \in \mathbf{R}^{(b+1) \times 1}$ such that:

$$
\begin{aligned}
& \left(\begin{array}{cc}
A_{f}^{T} P_{f} A_{f}-P_{f} & A_{f}^{T} P_{f} B_{f} \\
B_{f}^{T} P_{f} A_{f} & B_{f}^{T} P_{f} B_{f}
\end{array}\right)+\left(\begin{array}{c}
C_{f}^{T} \\
D_{f}^{T}
\end{array}\right) \mathcal{X}_{f}\left(\begin{array}{ll}
C_{f} & D_{f}
\end{array}\right) \leq 0 \\
& \left(\begin{array}{cc}
P_{\lambda}-A_{\lambda}^{T} P_{\lambda} A_{\lambda} & C_{\lambda}^{T}-A_{\lambda}^{T} P_{\lambda} B_{\lambda} \\
C_{\lambda}-B_{\lambda}^{T} P_{\lambda} A_{\lambda} & D_{\lambda}+D_{\lambda}^{T}-B_{\lambda}^{T} P_{\lambda} B_{\lambda}
\end{array}\right) \geq 0
\end{aligned}
$$

Here $\left(A_{\lambda}, B_{\lambda}, C_{\lambda}, D_{\lambda}\right)$ is as $(A, B, C, D)$ in Theorem 2 but with $m$ and $R_{r}(i)$ replaced by $b$ and $\lambda_{i}$, respectively. $\left(A_{f}, B_{f}, C_{f}, D_{f}\right)$ is a state-space representation of $\mathcal{F}(z)$ :

$\mathcal{F}(z)=\left(\begin{array}{c}\left(I_{5} \otimes \mathcal{B}\right) \Omega(z) \\ I_{k+1} \otimes \mathcal{B} \\ I_{k+1}\end{array}\right) \quad$ and

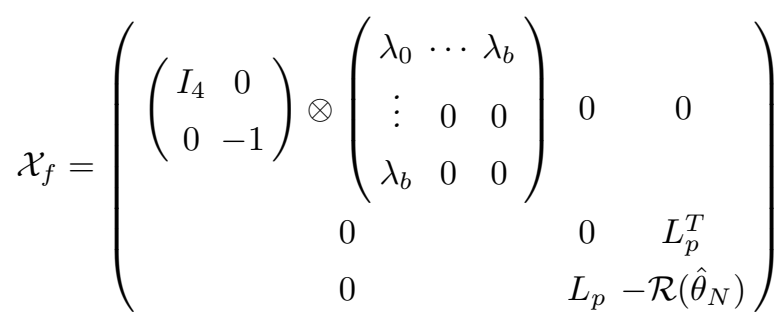

Proof. First note that, via some algebraic manipulations, constraint (18) can be rewritten as $\mathcal{F}^{*}\left(e^{j \omega}\right) \mathcal{X}_{f} \mathcal{F}\left(e^{j \omega}\right) \leq 0$ if one defines, in $(18), \tau(\omega)=$ $\lambda_{0}+\sum_{i=1}^{b} \lambda_{i}\left(e^{j i \omega}+e^{-j i \omega}\right)$ and $j \mathcal{L}(\omega)=L\left(e^{j \omega}\right)+L^{*}\left(e^{j \omega}\right)$ where $\bar{L}(z)=L_{p}\left(I_{k+1} \otimes \mathcal{B}(z)\right)$ is a matrix of FIR transfer functions such that $L(z)=-L(z)^{T}$. Second, note that this expression of $j \mathcal{L}(\omega)$ has the structure of Proposition 3 and that $\tau(\omega)$ is ensured to be a positive scalar at each $\omega$ via (B.1). Indeed, $\tau(\omega)=\lambda_{0}+\sum_{i=1}^{b} \lambda_{i}\left(e^{j i \omega}+e^{-j i \omega}\right)>0 \forall \omega$ is ensured by the existence of a symmetric matrix $P_{\lambda}$ such that (B.1) holds (see [16]). In this context, the existence of $\lambda_{i}(i=0 \ldots b)$ and of a matrix $L_{p}$ such that $\mathcal{F}^{*}\left(e^{j \omega}\right) \mathcal{X}_{f} \mathcal{F}\left(e^{j \omega}\right) \leq 0$ implies that $\bar{J}\left(\omega, G, \hat{C}, W_{l}, W_{r}\right) \leq 1, \forall G \in \mathcal{D}\left(\hat{\theta}_{N}, P_{\theta}\right)$. The result of the proposition follows then from a simple application of the KYP Lemma [19]. 